\title{
Non-invasive regime for language lateralization in right- and left-handers by means of functional MRI and dichotic listening
}

Received: 11 April 2001 / Accepted: 26 February 2002 / Published online: 8 May 2002

(C) Springer-Verlag 2002

\begin{abstract}
Language lateralization was assessed by two independent functional techniques, fMRI and a dichotic listening test (DLT), in an attempt to establish a reliable and non-invasive protocol of dominance determination. This should particularly address the high intraindividual variability of language lateralization and allow decisionmaking in individual cases. Functional MRI of word classification tasks showed robust language lateralization in 17 right-handers and 17 left-handers in terms of activation in the inferior frontal gyrus. The DLT was introduced as a complementary tool to MR mapping for language dominance assessment, providing information on perceptual language processing located in superior temporal cortices. The overall agreement of lateralization assessment between the two techniques was $97.1 \%$. Conflicting results were found in one subject, and diverging indices in ten further subjects. Increasing age, non-familial sinistrality, and a non-dominant writing hand were identified as the main factors explaining the observed mismatch between the two techniques. This finding stresses the concept of an intrahemispheric distribution of language function that is obviously associated with certain behavioral characteristics.
\end{abstract}

Keywords fMRI · Dichotic listening · Language lateralization · Left-handedness · Age effects · Gender

\section{Introduction}

The determination of language dominance has become an important issue in the presurgical benefit-risk evaluation of patients with brain tumors in eloquent areas and/ or intractable epilepsy (Loring et al. 1990; Gerschlager et al. 1998; Hajek et al. 1998). An adequate evaluation demands techniques that are reliable, reproducible, re-

M. Hund-Georgiadis $(-$ U. Lex $\cdot$ A.D. Friederici

D.Y. von Cramon

Max-Planck-Institute of Cognitive Neuroscience,

Stephanstrasse 1, 04103 Leipzig, Germany

e-mail: hund@cns.mpg.de peatable, and risk-free in nature, i.e., in the best case non-invasive. So far, the intracarotid amobarbital procedure (IAP; Wada and Rasmussen 1960) has been considered as the gold standard to assess language dominance, however, with major drawbacks. Its invasiveness provides an additional risk for the patient. Moreover, IAP cannot easily be repeated and the examination time is limited to the time frame of 20-30 min, therefore only restricted aspects of language organization can be addressed.

Additionally, the spatial resolution of IAP is very poor. As the technique is based on behavioral testing of certain language functions during anesthesia of one hemisphere, it can solely characterize language subfunctions within the whole hemisphere. Although the procedure was initially used to lateralize language function, it has further evolved to include prediction of degrees of verbal memory decline following left temporal lobectomy. The reliability of IAP has recently been questioned, when language lateralization is solely and primarily based on aspects of speech production such as speech arrest (Benbadis et al. 1998). However, IAP procedure focusing on language comprehension as described by Loring and coworkers (1990) is able to assess language lateralization as a more fine grained variable rather than a discrete category, such as 'left', 'right', or 'mixed'. The individual lateralization scores indicate a relative interhemispheric distribution of language functions with different contributions from various eloquent areas, although, without revealing their anatomical localization within the language network. Two main questions arise. First, what is the contribution of different eloquent cortices of both hemispheres to the processing of language? The answer is of interest in itself and leads straightforwardly to the second set of questions, namely, what are the clinical implications and applications of this line of inquiry? If language lateralization is a rather distributed effect, how does this knowledge affect surgical decisionmaking in individual patients and how does it influence reorganization of language function following stroke or hemorrhage in eloquent cortices? What is the clinical 
outcome of patients with mixed dominance? Thus it appears that there are many reasons to thoroughly examine the concept of distributed language dominance in normal right- and left-handed controls rather than applying it to patients. Due to methodological and technical limitations, IAP cannot sufficiently address this new research line.

A major effort has been made in precedent functional imaging research to replace the IAP by non-invasive fMRI mapping. Several comparative studies showed a reasonable agreement of the two techniques (Demb et al. 1995; Desmond et al. 1995; Binder et al. 1996). However, sole reliance on the alternative technique for identification of dominant eloquent cortices does not seem to be considered routinely as adequate and sufficient in the clinical setting. There are also some caveats in using fMRI-assessed lateralization data as a basis for surgery. First, lateralization is purely based on activated pixels in predefined eloquent cortices and does not necessarily relate directly to behavioral data. Second, to isolate the relevant cognitive process, the MR design is based on a comparison of the relevant target task with an appropriate baseline condition. Even slight changes of the experimental protocol may significantly influence the outcome, i.e., the lateralization index, and thereby may complicate clear functional decision-making. Third, almost all fMRI lateralization studies report contributions of the subdominant hemisphere, that have not been expected to this extent on the basis of either IAP and lesion studies. The role of the subdominant cortices in the overall language network identified by fMRI studies has to be further evaluated in future studies, beyond the sole determination of laterality. Answers to these issues are needed before the role of fMRI in the lateralization of language function can be fully determined.

Previous fMRI research has applied various paradigms to assess the dominant language site of the brain, in particular the fronto-opercular cortices. Much weight has been put on finding both the relevant target and the appropriate baseline task controlling for non-relevant cognitive processes. Word and sentence production tasks (Hertz-Pannier et al. 1997) as well as word classification tasks (Desmond et al. 1995; Gabrieli et al. 1995; Binder et al. 1996) have been used for this purpose. The activation in the inferior prefrontal gyrus (BA45, 46, 47) during lexical and semantic encoding tasks has been claimed to define global hemispheric dominance of speech. It is intriguing that the choice of the cut-offs for the categories 'left', 'right', and 'bilateral' was found to influence the statistical distribution of lateralization.

Moreover, different genetic, developmental, and environmental factors may influence the functional organization patterns of language as assessed by means of fMRI. So far, there are only vague ideas about the influence of age (Ross et al. 1997; D'Esposito et al. 1999), as most functional mapping studies are based on young control subjects. It has been claimed that a change of the hemodynamic coupling of the blood oxygenation level dependent (BOLD) to the neural response may accompany ag- ing. Moreover, there are contradictory statements about the influence of sex (Shaywitz et al. 1995; Frost et al. 1999; Springer et al. 1999). While Shaywitz et al. (1995) described a more bilateral dominance distribution in female healthy controls, Springer et al. (1999) and Frost et al. (1999) did not find any significant sex differences and argued against substantive differences between men and women in the large-scale neural organization of language processes.

Although the influence of hand dominance on the cerebral organization of language has been widely examined, it remains poorly understood. The influence of certain cofactors of left-handedness, such as familial sinistrality, inverted writing, or ambidextrous writing on the cerebral organization patterns is unclear. No doubt, these characteristics may contribute to a large intersubject variability, thereby complicating functional decisionmaking. If functional mapping research aims at routinely replacing IAP, a clear regime is required to deal with intraindividual inconsistencies and deviations.

Individual variability seems to pose an intriguing problem. Within the ever-expanding language mapping studies, fMRI data of normal control cohorts were recently collected (Springer et al. 1999). They reflect the expected distribution of language dominance in normal controls and show reasonable agreement with IAP. The single case though, especially when pathological brain structures are involved, may sometimes allow ambiguous and contradictory interpretations. Hence, definite determinations based on fMRI would profit from a 'second opinion', which should be acquired by other non-invasive behavioral tests or an extended MR protocol.

In the years since Kimura's initial reports (1967), the dichotic language test (DLT) has gained wide usage in both clinical and experimental settings. The direction of ear advantage (left versus right) is generally accepted as an indicator of the hemisphere which is superior for speech discrimination. The DLT thus enlightens a restricted aspect of language processing. The auditory discrimination at the word level is supposed to engage components of the language network related to perception and has been demonstrated to activate the superior temporal gyrus of both hemispheres to a different extent (Lee et al. 1994; Hugdahl et al. 1999). In contrast, the currently used fMRI lateralization paradigms are designed to activate inferior frontal cortices, which are more dedicated to language production. Comparison between DLT- and IAP-based language lateralization disclosed a high correlation in both the overall and the inbetween test results (Strauss et al. 1987; Zatorre 1989).

In the present study, language lateralization was assessed by both fMRI and DLT to evaluate the agreement of these non-invasive techniques. The issue of intersubject variability was particularly addressed, seeking to provide a diagnostic strategy appropriate to functional decision-making in individual subjects. It aims at characterizing the functional organization patterns of a relevant aspect of language in a group of left-handed subjects (LH) in comparison to right-handed subjects (RH), tak- 
ing into account the influences of sex, age, hand dominance, and familial sinistrality.

\section{Materials and methods}

Thirty-four healthy control subjects (18 male, 16 female), $17 \mathrm{RH}$ and $17 \mathrm{LH}$, aged from 20 to 67 years $(32.4 \pm 15.87)$ were included in the study. Eight out of 17 LH had a history of familial sinistrality. Nine subjects were ambidextrous. All participants had normal or corrected to normal vision. To examine age-dependent lateralization effects, 10 subjects ( 5 male, 5 female) aged over 40 years were included in the study. The group statistics was performed for the entire groups of RH and $\mathrm{LH}$, in a second step for the two age subgroups (G1, age <40 years; G2, age > 40 years), and in a third step separately for males and females. The experiments were generally approved by the local ethics committee, and all subjects gave written informed consent to participate in the study.

\section{Behavioral tasks}

Each subject performed the Edinburgh handedness inventory (EHI) to assess the degree of handedness (Oldfield 1971).

Speech lateralization was determined in behavioral terms using an auditory dichotic listening test based on the discrimination of rhyme words (DLT; Diesch and Haettig 1997). In the task ten monosyllabic pairs of rhyme words were simultaneously presented to the left and the right ear. The subjects were asked to loudly repeat the word which they had identified. A lateralization index (LI) was calculated from the number of detected right $\left(\mathrm{n}_{\mathrm{Pr}}\right)$ and left ear stimuli $\left(\mathrm{n}_{\mathrm{Pl}}\right)\left[\mathrm{LI}=\mathrm{n}_{\mathrm{Pr}}-\mathrm{n}_{\mathrm{Pl}} / \mathrm{n}_{\mathrm{Pr}}+\mathrm{n}_{\mathrm{Pl}}(\right.$ range \pm 1$\left.)\right]$. Positive values indicate right ear and left-hemispheric dominance. Bilaterality was defined when values ranged between \pm 0.1 . The DLT is based on the principle that, whenever two auditory signals are put in conflict, the contralateral input is suppressing the ipsilateral signal. Therefore, the ear contralateral to the dominant language cortex should show an advantage over the ipsilateral ear.

\section{Activation paradigms}

During the fMRI experiment, common German words composed of two syllables (length: four to seven letters) were visually presented every $2 \mathrm{~s}$ in a word classification task. The target stimuli consisted of nouns and verbs, half abstract and half concrete, divided in eight blocks of 20 words each, with a pseudorandom presentation order. Semantic (abstract or concrete) encoding was compared to a perceptual task (presentation of words with normal or double spacing of letters). The lexical encoding task (noun or verb) was contrasted with a different perception task (presentation of words in capital or small letters). The baseline condition was either one of the two perceptual encoding conditions or a resting condition (dark desktop consisting of five white Xs presented for $40 \mathrm{~s}$ ). The order of the MR scanning protocol varied between subjects and started randomly with the lexical encoding or with the semantic encoding tasks. The baseline condition task, i.e., perceptual encoding versus resting condition constituted the last part of the scanning protocol. All applied word stimuli were presented only once within the overall protocol to counteract effects of task priming. During the fMRI experiment behavioral data (reaction times and number of errors) were recorded online.

\section{Functional MRI recording}

Functional MRI recording was performed on a 3T whole body system (Medspec 300/100; Bruker, Ettlingen, Germany) using EPI (seven axial slices, $7 \mathrm{~mm}$ slice thickness, TE=30 ms, TR=2 s, matrix $128 \times 64$, field of view $25 \mathrm{~cm}$ ).
Image analysis

Functional MRI data were analyzed with the BRIAN Software (Kruggel and Lohmann 1996). The initial four words target items were excluded from analysis to avoid influence of vascular arousal caused by the onset of the scanner noise. Preprocessing included movement correction in 2D, and baseline correction entailing filtering of functional data in the temporal domain by a lowpass filter. Functional images were created by generating statistical z-maps with z-values $>5$ on a single pixel level. The regions of interest were characterized in size, volume, and intensity of activity. Lateralization indices were calculated by the number of activated pixels $(\mathrm{P})$ with a $\mathrm{z}$-score higher than 5.5 in Broca's area [Talairach and Tournoux (1988): cC6, cC7, cD5, cD6, cD7], in the superior temporal gyrus (Talairach: $\mathrm{cF} 10, \mathrm{cF} 9, \mathrm{cG} 10, \mathrm{cG} 9, \mathrm{cE} 9, \mathrm{cE} 10$ ), and for each hemisphere $\left[\mathrm{LI}=\mathrm{n}_{\mathrm{Pl}}-\mathrm{n}_{\mathrm{Pr}} / \mathrm{n}_{\mathrm{Pl}}+\mathrm{n}_{\mathrm{Pr}}(\right.$ range \pm 1$\left.)\right]$. Lateralization indices were subsequently classified according to Strauss et al. (1987) as left hemisphere language dominant (defined as LI>0.1), symmetric $(-0.1 \leq \mathrm{LI} \leq 0.1)$, and right hemisphere dominant (defined as $\mathrm{LI}<-0.1$ ). In accordance with previous lateralization studies functional decision-making in individuals employed the Broca index (BI) as the main fMRI-based index. A temporal index (TI) was taken into account, when fMRI index and DLT index showed a deviation of more than $40 \%$. Individual data were transformed into the Talairach coordinate system and averaged within groups to separately assess the activation patterns of LH and RH.

Statistical analysis and group comparisons were conducted using computerized statistical software (version 5.01; SPSS, Chicago, Ill., USA). Normally distributed data were expressed as mean \pm SD. Independent $t$-tests were used to test group differences. Significance was declared at the $P<0.05$ level. To assess the relationship between the LIs acquired using the two different techniques, bivariate correlation analysis using Pearson's coefficient was performed. The LI scores were subjected to a stepwise discriminant analysis procedure (Hermans et al. 1982), where 'natural subgroups' were generated based on the LIs. The program allows a stepwise forward selection of best discriminating variables. By using the leaving-one-out method, the program also prevents an underestimation of the error of misclassification.

\section{Results}

\section{Behavioral data}

The EHI evaluation showed mean values of $0.84 \pm 0.14$ and $-0.67 \pm 0.26$ for $\mathrm{RH}$ and $\mathrm{LH}$, respectively. The EHI results were in accordance with the individual history of handedness in all subjects. The DLT disclosed mean lateralization indices of $0.59 \pm 0.37$ and $0.02 \pm 0.55$ for $\mathrm{RH}$ and $\mathrm{LH}$, respectively (maximum values: -1 for right and +1 for left-hemispheric dominance. A hemispheric dominance was declared when the lateralization index differed more than $10 \%$ from zero (Strauss et al. 1987). This was the case in 16 out of $17 \mathrm{RH}$ (94\%), in whom left hemisphere language dominance was diagnosed. In the group of LH, 8 subjects were classified as left hemisphere dominant (47\%), 2 as bilateral (12\%), and $7(41 \%)$ as right hemisphere language dominant. The correlation between EHI and DLT was high $(r=0.52, P=0.002)$.

\section{Functional MRI-encoding paradigms}

Analysis of performance during the word classification tasks showed mean reaction times of $702 \pm 67 \mathrm{~ms}$ in the 
Fig. 1 Group analysis of the semantic encoding task versus perceptual encoding for lefthanders $(n=17)$ and right-handers $(n=17)$. A mean $\mathrm{z}$-score of z>3 was chosen to define relevant activation. The averaged and normalized group data are overlaid on a reference $3 \mathrm{D}$ dataset. The task-related functional activations were located in the inferior frontal gyrus extending to the middle frontal gyrus corresponding to BA44/45 and BA6. A clear preponderance of the left hemisphere was found in right-handers, while bilateral activation patterns were assessed in the left-handers

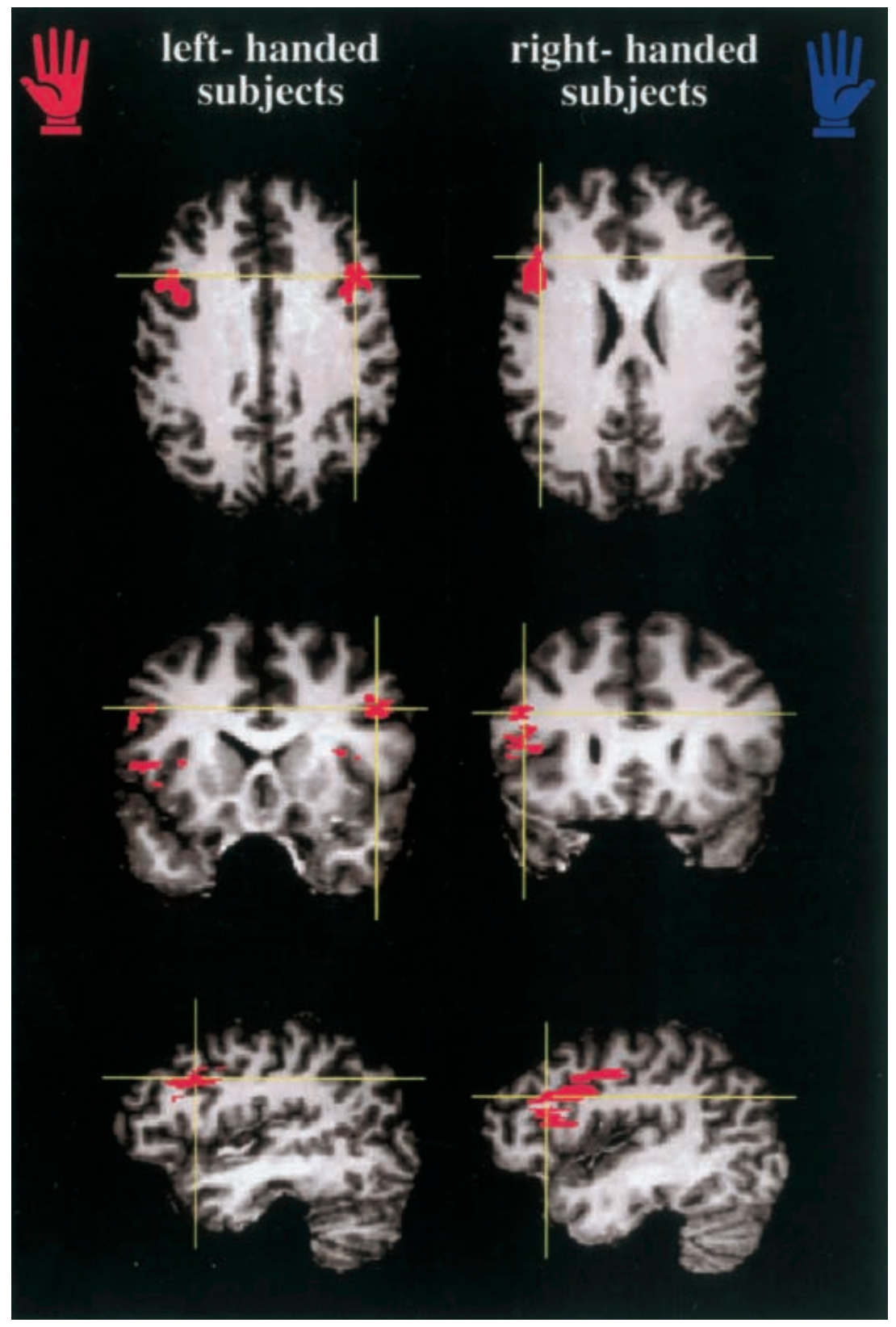

G1 group with an error rate of $23 \%$ and of $717 \pm 68 \mathrm{~ms}$ (error rate $33 \%$ ) in the G2 group (all differences non-significant). Functional MRI data analysis showed extended activation of the inferior frontal gyrus (pars triangularis) during the semantic and lexical encoding conditions in all studied subjects. The clearest lateralization effects were found during semantic encoding when the perceptual condition was applied as baseline. However, more bilateral activation was observed when fixation served as the baseline condition. Figure 1 displays the functional group data for both LH and RH. Table 1 gives the Talairach coordinates and the overall amount of activation in Broca's area during semantic encoding against the perceptual encoding condition.
Other encoding conditions

The lexical encoding task yielded similar activation patterns as semantic encoding, when perceptual encoding was applied as baseline (Table 2). However, the quantitative and qualitative magnitude of the MR signal changes was smaller as compared to the semantic encoding condition. When fixation was chosen as baseline condition, activation extended to further anatomical structures such as medial frontal gyrus, superior frontal gyrus, anterior insula, praecentral gyrus, central and postcentral gyrus, supramarginal gyrus, medial temporal gyrus, thalamus, and basal ganglia. The same observation was made in the semantic encoding task against fixation baseline. In sum, the different encoding tasks had a differential potential to detect laterality. For clarification, the resulting mean BIs 
Table 1 Talairach coordinates and volumes $\left(\mathrm{mm}^{3}\right)$ of the main activations in eloquent cortices $(z>3)$ separately listed for right-handers (RH) and left-handers (LH) during semantic versus perceptual encoding. (GFI Inferior frontal gyrus, GFM medial frontal gyrus)

\begin{tabular}{|c|c|c|c|c|}
\hline \multirow[b]{2}{*}{ Area } & \multicolumn{2}{|c|}{ Talairach coordinates } & \multicolumn{2}{|c|}{ Volumes (z-max) } \\
\hline & Left & Right & Left & Right \\
\hline \multicolumn{5}{|l|}{$\mathrm{RH}(n=17)$} \\
\hline GFI (BA44) & -421127 & --- & $1,522(3.98)$ & - \\
\hline Anterior insula & -37206 & --- & $164(2.87)$ & - \\
\hline \multicolumn{3}{|c|}{ Total volume $\left(\mathrm{mm}^{3}\right)$} & 1,686 & - \\
\hline \multicolumn{5}{|l|}{$\mathrm{LH}(n=17)$} \\
\hline GFI (BA44) & -36130 & 35332 & $395(2.90)$ & $208(2.66)$ \\
\hline GFI (BA45) & -421716 & 432420 & $647(2.82)$ & $132(2.56)$ \\
\hline GFM (BA46/9) & --- & 352727 & - & $131(2.49)$ \\
\hline Anterior insula & -39210 & --- & $30(2.26)$ & - \\
\hline \multicolumn{3}{|c|}{ Total volume $\left(\mathrm{mm}^{3}\right)$} & 1,072 & 471 \\
\hline
\end{tabular}

Table 2 Talairach coordinates and volumes $\left(\mathrm{mm}^{3}\right)$ of the main activations in eloquent cortices $(z>3)$ separately listed for right-handers and left-handers during lexical encoding versus perceptual encoding

\begin{tabular}{|c|c|c|c|c|}
\hline \multirow[b]{2}{*}{ Area } & \multicolumn{2}{|c|}{ Talairach coordinates } & \multicolumn{2}{|c|}{ Volumes (z-max) } \\
\hline & Left & Right & Left & Right \\
\hline \multicolumn{5}{|l|}{ RH $(n=17)$} \\
\hline $\begin{array}{l}\text { GFI (BA44/6) } \\
\text { GFI (BA44/45) } \\
\text { Anterior insula } \\
\text { Thalamus }\end{array}$ & $\begin{array}{l}-47616 \\
-401615 \\
-2686 \\
-1-717\end{array}$ & $\begin{array}{l}--- \\
--- \\
--- \\
---\end{array}$ & $\begin{array}{r}11(1.59) \\
496(2.34) \\
72(1.78) \\
84(1.97)\end{array}$ & \\
\hline \multicolumn{3}{|c|}{ Total volume $\left(\mathrm{mm}^{3}\right)$} & 663 & \\
\hline \multicolumn{5}{|l|}{ LH $(n=17)$} \\
\hline $\begin{array}{l}\text { GFI (BA44/6) } \\
\text { GFI (BA44/45) } \\
\text { Anterior insula } \\
\text { Thalamus }\end{array}$ & $\begin{array}{llll}-44 & 5 & 15 \\
-40 & 19 & 13 \\
-30 & 20 & -1 \\
-7 & -19 & 15\end{array}$ & $\begin{array}{l}--- \\
--- \\
--- \\
---\end{array}$ & $\begin{array}{r}9(1.61) \\
120(1.90) \\
10(1.60) \\
84(1.76)\end{array}$ & \\
\hline \multicolumn{3}{|c|}{ Total volume $\left(\mathrm{mm}^{3}\right)$} & 213 & \\
\hline
\end{tabular}

of the various conditions are displayed in Fig. 2. As the semantic encoding condition showed the clearest lateralization effects, this condition was used for further statistical comparisons.

The perceptual control tasks (against fixation as baseline) did not yield relevant activation in language-related areas (i.e., BA44, 45, 46). Activation was found in the primary motor and sensory cortex and in premotor cortices (BA6), as expected.

Functional MRI-based lateralization of language function showed clearer lateralization effects in RH than in LH. Based on the fMRI-obtained BI, left-hemispheric language dominance was found in 16 out of $17 \mathrm{RH}$ with a mean lateralization index of $0.5 \pm 0.39$. One $\mathrm{RH}$ unexpectedly showed right-hemispheric dominance, which was in accordance with the DLT results. In the LH group, 9 subjects were classified as left dominant for

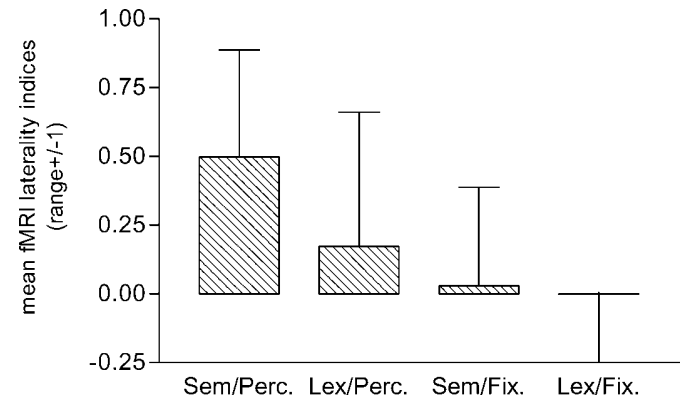

Fig. 2 Different potential of the applied word classification and baseline tasks to show laterality. The mean laterality indices of the various tasks are displayed for the group of right-handers. Semantic encoding contrasted with a perceptual control task was the best indicator of lateralization

language, 1 showed symmetric language organization, and 7 were defined as right dominant related to cortical language representation (BI for the entire group $0.19 \pm 0.59)$. Language dominance could definitely be determined in 33 out of 34 examined subjects $(97 \%)$ by means of fMRI. The more global hemispheric lateralization index (HI) yielded comparable results: HI was $0.52 \pm 0.34$ and $0.14 \pm 0.6$ in the RH and LH groups, respectively. A highly significantly correlation $(r=0.9$, $P<0.001)$ was found between the fMRI-gained indices $\mathrm{HI}$ and BI. Significant temporal activation (z-score >5.5) was assessed in 18 out of 34 subjects with mean TIs of $0.49 \pm 0.2$ and $0.07 \pm 0.4$ for $\mathrm{RH}$ and $\mathrm{LH}$, respectively. Statistically significant correlations were also found between TI and BI $(r=0.8, P<0.001)$ and between TI and $\mathrm{HI}(r=0.77, P<0.001)$.

It must be noted that, apart from two subjects, one $\mathrm{LH}$ and one $\mathrm{RH}$, all controls employed the subdominant hemispheres during the word classification task to a certain degree: a total of $33.4 \pm 17 \%$ of activation in $\mathrm{RH}$ was located the right hemisphere, while a total of $56.6 \pm 24 \%$ of activation in LH was located in the left hemisphere.

\section{Correlations with other behavioral tests}

The following correlations between fMRI and behavioral indices are based on the semantic encoding task versus perceptual condition: BI and DLT $(r=0.75, P<0.001)$, HI and DLT ( $r=0.51, P=0.016)$, and TI and DLT $(r=0.8$, $P<0.001)$. The distribution of lateralization indices based on DLT and fMRI for individual subjects is displayed in Fig. 3. In addition, Fig. 4 separately shows the group results for left- and right-handers. No significant correlation was found between: (1) the degree of handedness based on EHI and language lateralization based on fMRI, (2) the performance data and the lateralization indices, or (3) the performance data and the overall amount of activated pixel during the fMRI experiment. 
Fig. 3 Dichotic listening test (DLT)- and fMRI-obtained indices of individual subjects are shown, separately for left- and right-handers

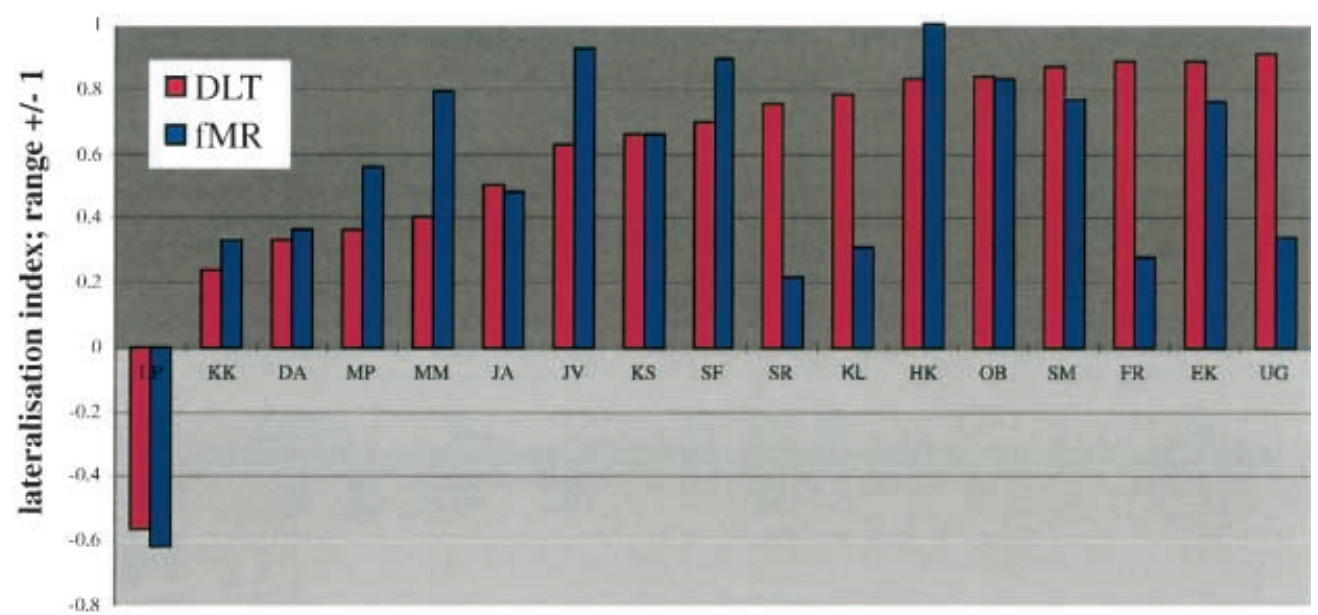

right-handed subjects

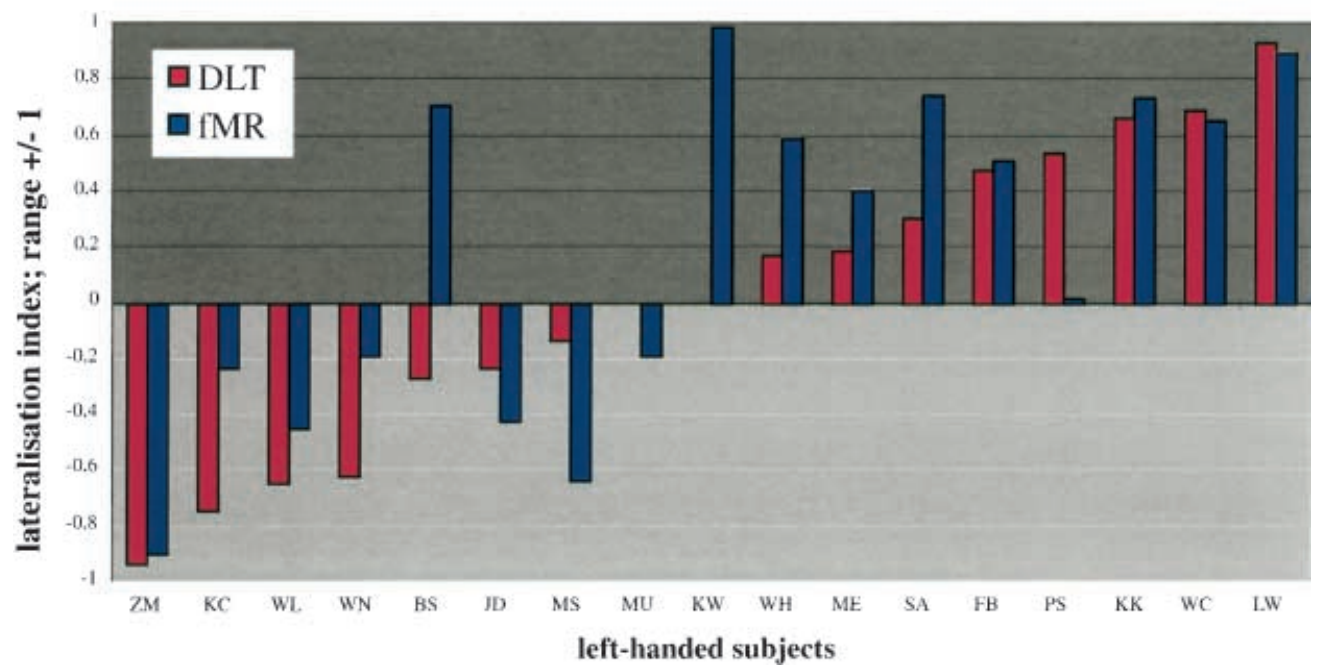

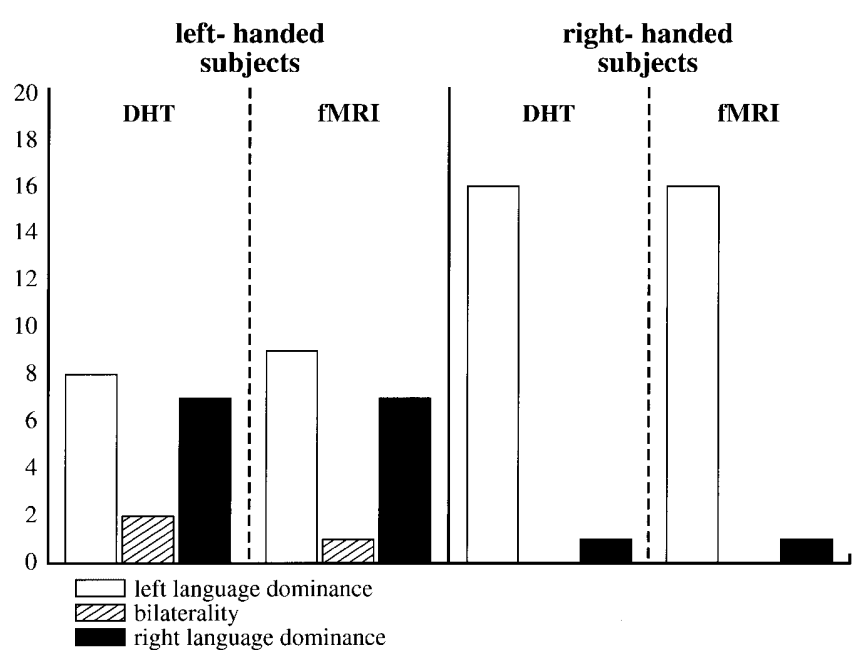

Fig. 4 Distribution of language dominance based on the two applied techniques, DLT and fMRI

\section{Discriminant analysis}

The first step of the analysis determined the proportion of controls that could be reliably identified as right- or left-handed based on DLT and fMRI LIs (Fig. 5A). The DLT-LI was the best discriminating variable related to handedness, as $79.4 \%$ of all subjects were correctly classified as left- or right-handers. In contrast, the correct classification rate of the variable 'fMRI-LI' was $71.5 \%$. However, when categorical left and right hemisphere language dominance as defined by DLT was introduced as group discriminator (Fig. 5B), as many as $94.1 \%$ of all subjects were correctly identified based on fMRI-LIs.

\section{Sex differences}

Gender did not play a decisive role in the organization patterns of language in our sample, as no significant differences were found when comparing the lateralization indices of female and male subjects (Table 3). This was true for both, fMRI and DLT results. Though there were no differences in definite language lateralization, the em- 

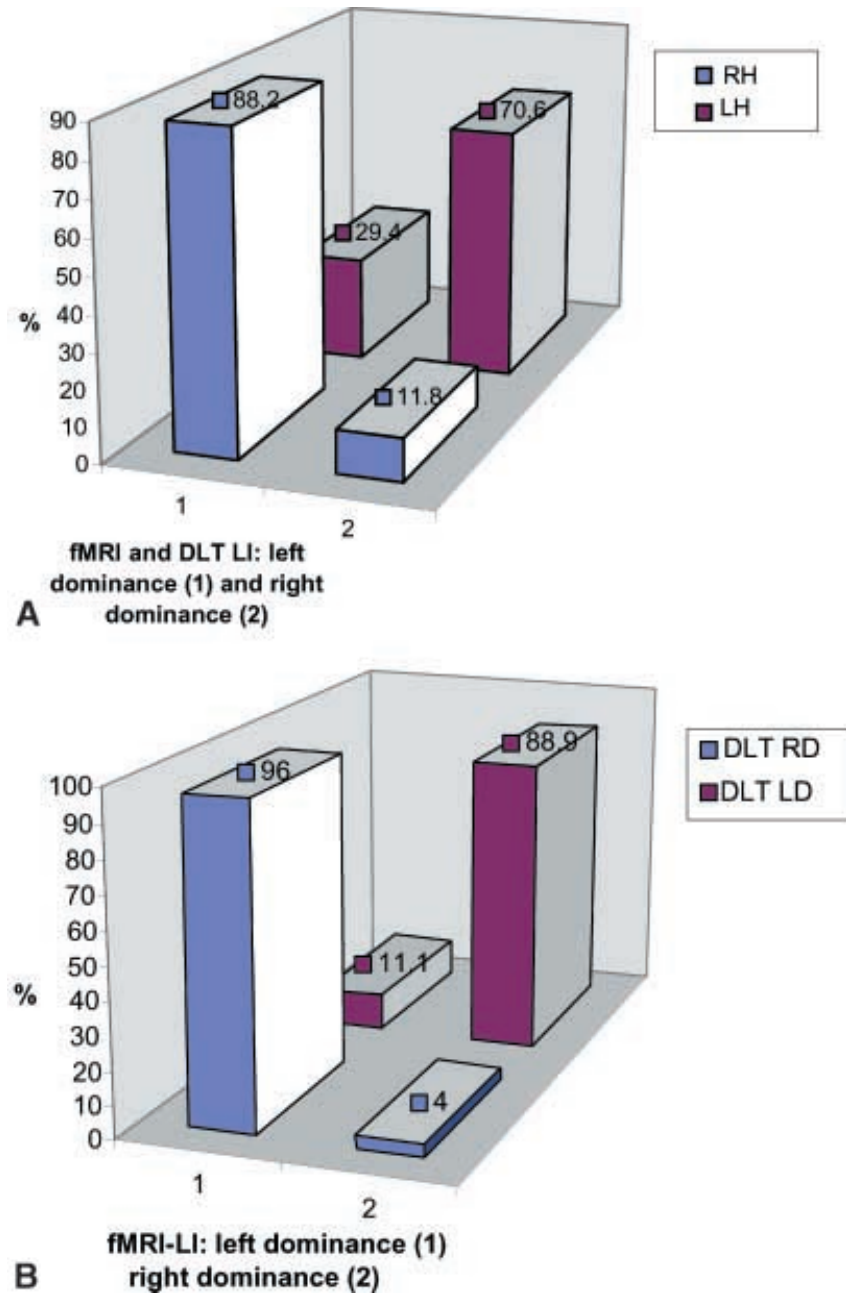

Fig. 5A, B Classification of right- and left-handers according to a stepwise discriminant analysis procedure. In the first step of the analysis the proportion of controls was determined that could be reliably identified as right- or left-handed based on DLT- and fMRI-gained lateralization indices (LIs; A). Related to handedness the best discriminating variable was the DLT-LI; 79.4\% of all subjects were correctly classified as left- or right-handers. When categorical left and right hemisphere language dominance as defined by DLT was introduced as group discriminator (B), as many as $94.1 \%$ of all subjects were correctly identified based on fMRI-LIs

Table 3 Mean Dichotic listening test (DLT) and fMRI-based lateralization indices separately displayed for male and female subjects

\begin{tabular}{lrl}
\hline & \multicolumn{1}{l}{ DHT } & fMRI \\
\hline RH male & $0.45 \pm 0.47$ & $0.37 \pm 0.47$ \\
RH female & $0.71 \pm 0.29$ & $0.62 \pm 0.26$ \\
LH male & $-0.03 \pm 0.55$ & $0.17 \pm 0.57$ \\
LH female & $0.09 \pm 0.59$ & $0.19 \pm 0.68$ \\
\hline
\end{tabular}

ployed anatomical regions slightly differed in female and male subjects during semantic encoding: while the group analysis of females showed recruitment of areas BA44 and BA6, there was an additional involvement of the anterior insula in males.

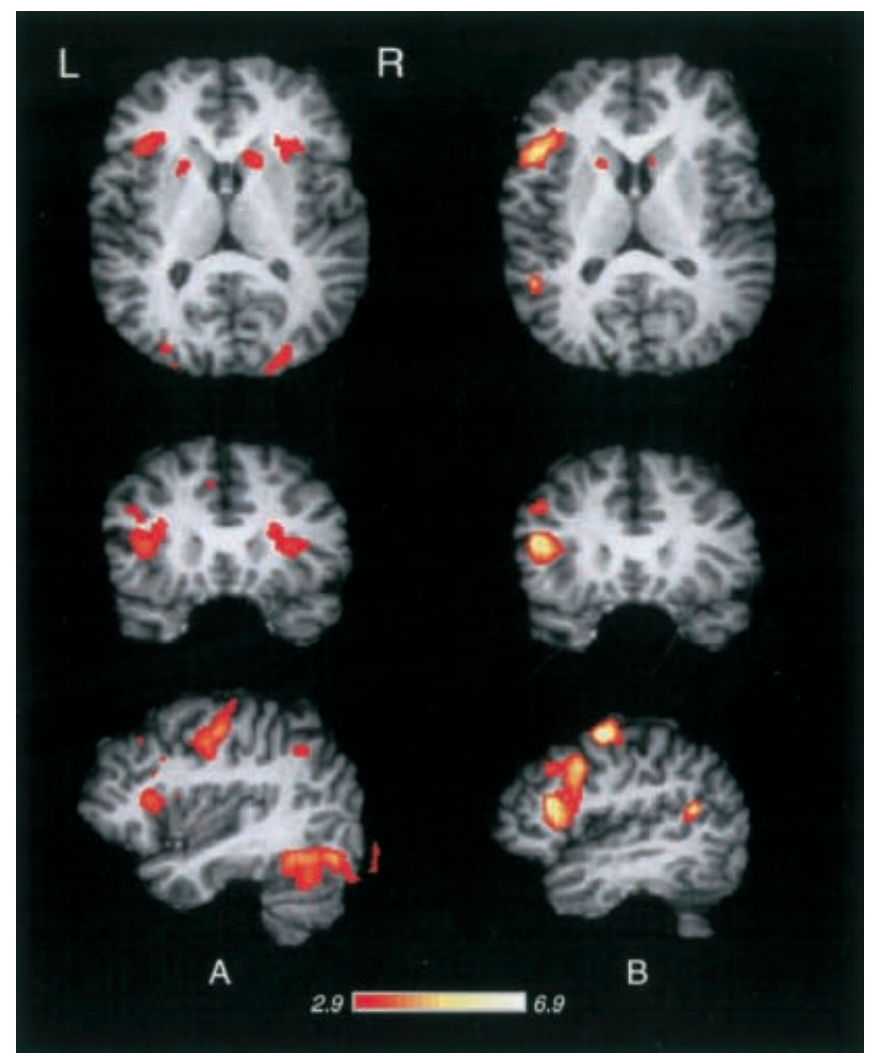

Fig. 6 Group data of familial left-handers (A) and non-familial left-handers (B) in the stereotactic space. Significantly lower LIs in the familial group indicated a tendency to clearer lateralize language to the right hemisphere in this group. The task-related activation was again found mainly in BA44. Noteworthy, familial lefthanders with more bilateral activation patterns in the inferior frontal gyrus as well showed bilateral activations in basal ganglia

\section{Familial sinistrality}

The LH groups with and without a history of familial sinistrality did differ significantly in test results and LIs (Fig. 6). The most pronounced difference was observed in the fMRI-based BI with indices of $-0.18 \pm 0.56$ and $0.44 \pm 0.49$ for familial $(n=8)$ and non-familial LH $(n=9)$, respectively $(P=0.04)$. Similar results were obtained from the DLT: $-0.29 \pm 0.38$ and $0.23 \pm 0.46$ for familial and non-familial LH, respectively $(P<0.04)$. However, EHI indices did not significantly differ between the groups $(-0.77 \pm 0.16$ for familial and $0.61 \pm 0.3$ for nonfamiliar LH). Hence, familial LH was associated with a significantly higher amount of right-hemispheric language lateralization as opposed to non-familial LH.

\section{Age differences}

Analysis of age-dependent lateralization effects showed a clearer language dominance in the G1 as compared to the G2 group, resulting in higher LIs. This was true for both RH and LH; the different laterality indices are sum- 
Table 4 Mean DLT and fMRI-based lateralization indices separately displayed for the two age groups: G1 ( $<40$ years) and G2 (>40 years)

\begin{tabular}{lcl}
\hline & \multicolumn{1}{l}{ DHT } & fMRI \\
\hline RH $<40$ years $(n=12)$ & $0.65 \pm 0.25$ & $0.65 \pm 0.27$ \\
RH $>40$ years $(n=5)$ & $0.35 \pm 0.57$ & $0.20 \pm 0.45$ \\
LH $<40$ years $(n=12)$ & $-0.1 \pm 0.57$ & $0.11 \pm 0.57$ \\
LH $>40$ years $(n=5)$ & $0.3 \pm 0.43$ & $0.37 \pm 0.68$ \\
\hline
\end{tabular}

marized in Table 4. The DLT and fMRI-based indices showed corresponding results. Subjects of the G2 group employed the same brain areas as the G1 during semantic encoding, but showed higher amounts of overall activation with a more extended bilateral involvement. While the amount of overall activated pixel $\left(\mathrm{P}_{\mathrm{n}}\right)$ was $84,203 \pm 60,002$ in the G1 group (left hemisphere: $61.5 \%$, right hemisphere: $38.5 \%), \mathrm{P}_{\mathrm{n}}$ in the $\mathrm{G} 2$ group was 12,4410 $\pm 72,523$ (left hemisphere: $54.3 \%$, right hemisphere: $55.7 \%)$.

Individual variability of lateralization assessment

In a further approach, data analysis concentrated on the individual assessment of language dominance based on fMRI and DLT. A mismatch of dominance determination was found in 11 subjects, $4 \mathrm{RH}$ and $7 \mathrm{LH}$. This was based: (1) on opposite values of the DLT and Broca LIs, indicating contradictory dominance $(n=1, \mathrm{LH})$, or (2) on a difference between the two indices exceeding 0.4 ( $n=10$; RH $n=4$, LH $n=6)$. Three of 4 RH (75\%) and 5 of $7 \mathrm{LH}(62.5 \%)$ exhibiting a mismatch in dominance determination belonged to the G2 group. Furthermore, all LH with mismatching indices were trained to write with the subdominant right hand and moreover belonged to the group of non-familial left-handedness. Thus, the factors age, non-familial sinistrality, and writing hand were associated with an intrahemispheric distribution of language dominance.

To determine language lateralization in these mismatch cases the MR data were further evaluated. In addition to the BI, TI was calculated in each individual subject. Hence, a clear language determination was declared when TI, as a measure of language perception rather than language production, was compensating the mismatch of DLT and BI by delivering a value in the range of one of these two indices. Following this analysis, a definite lateralization was achieved in three out of four mismatched $\mathrm{RH}$; no significant temporal activation was present in the remaining RH subject. Furthermore, in the mismatch group of LH seven out of eight subjects were clearly classified based on the additional TI, whereas no sufficient temporal activation was present in one subject. Figure 7 displays the three indices of the mismatch group.

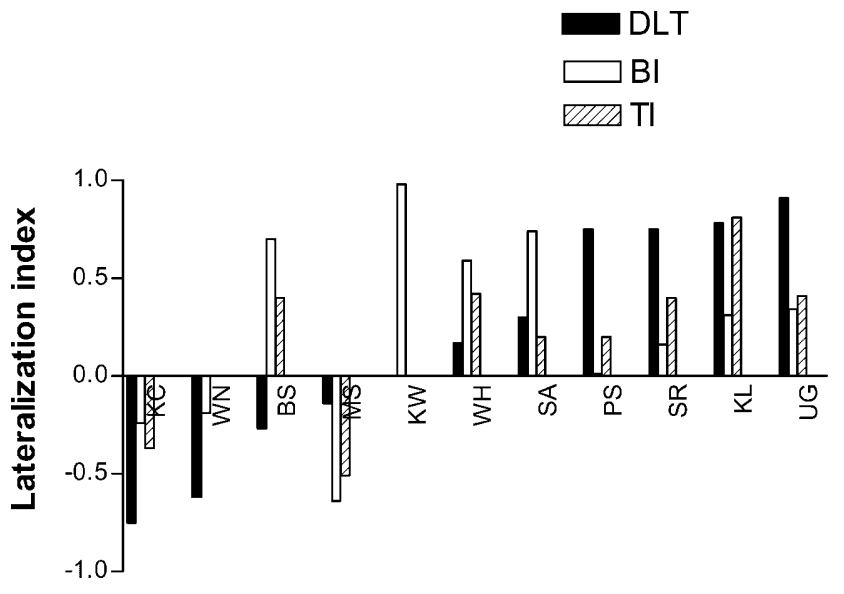

Fig. 7 Lateralization indices of all mismatch cases: DLT, Broca indices (BI), and temporal indices (TI) are displayed for the individual subjects. If significant temporal activation was assessed, the TI ranged between DLT and BI values

\section{Discussion}

Two independent functional techniques, fMRI and DLT, were employed to assess language lateralization in this study. Both techniques have been separately evaluated in previous lateralization research. The underlying goal of our investigation was to establish a reliable and noninvasive protocol of dominance determination, particularly addressing the high intraindividual variability of language lateralization in different paradigms and thus providing an appropriate tool for decision-making in individual cases.

\section{Dominance assessment by fMRI}

The word classification tasks were shown to be able to lateralize language function, when employed as a target task against a perceptual encoding control condition. The cerebral network supporting visual processing of language at the single word level mainly included the classical Broca area (BA44, 45) and, to a lesser degree, the homotopical inferior frontal gyrus in the non-dominant as well as the superior temporal gyrus in the dominant hemisphere. Of all RH, 94\% showed left-hemispheric language lateralization. In the LH group, $53 \%$ of subjects were classified as left hemisphere dominant, $6 \%$ as bilateral, and $41 \%$ as right hemisphere language dominant. Our results are in accordance with a series of fMRI studies that defined laterality of language function by means of inferior frontal gyrus activation obtained through different language tasks (Desmond et al. 1995; Gabrieli et al. 1995).

The semantic encoding task (versus perceptual encoding) provided the strongest fMRI activation of the classical Broca area and the clearest assessment of language dominance. Different baseline conditions were associated with more bilateral activation and even with the en- 
gagement of voxel in other non-eloquent brain areas. The lexical encoding task (versus perceptual encoding) requiring a decision about the item's word class (noun or verb) employed both eloquent cortices, however with a reduced magnitude of the corresponding BOLD signal change. This might be due to the fact that classification of nouns and verbs was associated with syntactic aspects of language processing. The observation that syntactic processing, in contrast to processing on the single word level, is associated with more bilateral inferior frontal gyrus activation was already described in a previous fMRI study from our institution (Ferstl and von Cramon 2000). In contrast, semantic encoding necessitates recognition of the word and retrieval of the whole word context to accomplish the abstract/concrete judgment. As the fMRI lateralization indices may significantly change depending on different target or control tasks, identification of the relevant cognitive system requires both an appropriate target task and a baseline task to control for nonspecific neural processes. Isolation of the relevant process strongly depends on the degree to which the two tasks accomplish their intended purposes. Hence, with respect to our task design, implementation of a resting stage as control task would not be sufficient to isolate the relevant cognitive process.

\section{Dominance assessment by DLT}

Left-hemispheric dominance was assessed in all but one right-handed subject. In contrast, the LH group showed an almost symmetrical distribution of LIs. Moreover, the LH had lower LI values, which indicate a less prominent lateralization. Our results are in accordance with several studies employing DLT, as summarized by Hongkeun (1994). Similar to the IAP, DLT is assessing language dominance as a result of a behavioral test. The DLT technique applied in the present study is clearly stimulusdriven according to Hugdahl (2000). Hence, it assesses the specialization between the hemispheres for processing of certain verbal stimuli in the absence of instructional interactions. Given the behavioral character of the test, we can only speculate about the underlying involvement of the temporal language cortices according to Hugdahl et al. (1999).

\section{Comparison of DLT and fMRI}

Based on analysis of LIs obtained by means of fMRI and DLT, the dominant site could clearly be assessed in 33 out of 34 subjects. The DLT and fMRI results were conflicting in one LH, in whom fMRI had attributed leftand DLT right-hemispheric dominance. Furthermore, the degree of lateralization obtained from the two techniques differed in 11 subjects ( $4 \mathrm{RH}$ and $7 \mathrm{LH}$ ) by more than $40 \%$. Two subjects were classified with bilateral language organization by the DLT technique, as opposed to 1 subject by fMRI.
What are the reasons for this mismatch between the two techniques? And more importantly, do we have to return to the IAP procedure to obtain clearer results for functional decision-making in patients? Both techniques, fMRI and DLT, differently approach the goal of dominance assessment. The classical Broca area was activated during the fMRI word classification task in all subjects, while additional temporal activation was only was found in 18 study participants. The activation of the inferior frontal gyrus is primarily associated with speech production within the language network. In the present fMRI word classification task these areas may be strongly activated, as subjects implicitly produce the visually perceived stimuli to assure good and rapid classification performance. Hence, the fMRI tasks clearly examine brain laterality from an instruction-driven perspective.

In contrast, the DLT reflects, as a stimulus-driven test, the limited capacity of the brain to handle two auditory stimuli presented exactly at the same time, one in each ear (Kimura 1967) and particularly when the two events draw on the same kinds of processing attributes. The DLT-based laterality is caused by the fact that although auditory input is transmitted to both auditory cortices in the temporal lobes, the contralateral projections are stronger and more preponderant, interfering with the ipsilateral projections. The advantage for the contralateral auditory projections means in a typical right-hander that the language dominant left hemisphere receives a stronger signal from the contralateral right ear. The DLT is hypothesized to involve perceptive components of the language network, represented in the superior and middle temporal gyrus. Hence, a mismatch between fMRI- and DLT-based laterality can have several explanations:

1. Laterality can be regarded as a combined variable of stimulus-driven and instruction-driven effects that dynamically interact in a given cognitive context. Therefore, individuals may express different laterality related to the stimulus modality.

2. Moreover, a mismatch of fMRI and DLT laterality can be interpreted as a reflection of an intrahemispheric distribution of language dominance rather than conflicting indices gained by two different techniques.

Evidence for a distributed intrahemispheric dominance regarding frontal and temporal cortices was as well found in a previous study by Lehéricy et al. (2000) who found strong evidence that task-related activations in frontal cortices were the clearest indicators of laterality and often were conflicting with corresponding activations in temporal cortices. Our observation that fMRIgained temporal LIs were ranging between BIs and DLT-LIs further supports this assumption. Hence, the combined protocol of fMRI and DLT enables the evaluation of different aspects of the language network, thus providing additional information to the examiner.

Discriminant analysis further supports the acceptable agreement between the two techniques, with a high reclassification rate for hemisphere language dominance of 
94.1\% when DLT was employed as group discriminator and fMRI-LIs as discriminating parameter.

\section{Cofactors of language lateralization}

The influence of genetic, developmental, environmental, and pathological factors on the distribution of language dominance has been addressed by many studies (Annett 1985, 1994; Buckner et al. 1995; Price et al. 1996; Springer et al. 1999). However, the significance of these influences remains contradictory and poorly defined. Our study adds weight to the hypothesis that gender, age, sinistrality, and writing hand influence the interhemispheric distribution of language dominance. No significant differences in the distribution of language lateralization were found in relation to gender. Though some different activation patterns were assessed within the inferior frontal gyrus, this was most probably related to the general interindividual variability of language lateralization, rather than gender differences. This finding is in conflict with observations of Shaywitz et al. (1995), who described a stronger left lateralization within the inferior frontal gyrus for the male, and a more bilateral organization in the female group. Shaywitz and coworkers employed a phonological decision task during their fMRI experiment. However, these researchers could not reproduce these differences using a word classification task, which is closer to our task design.

Age plays an important role in the distribution patterns of language dominance: lower BIs were observed in the G2 group, although the same anatomical areas were recruited during semantic encoding. These observations were probably influenced by two external factors. First, more motion artifacts were present in the G2 group. Second, the G2 subjects reported greater difficulties in performing the task as compared to the G1 subjects, although no significant difference in fMRI performance data was obvious between the two groups. The different functional patterns of the G2 group could also be due to an age-related change in the cortical network. This assumption is in accordance with the findings of Cabeza et al. (1997), who reported that encoding and retrieval processes tended to lateralize less clearly with increasing age of the subjects in their PET study. They hypothesized that these differences could be due to an overall functional reorganization co-occurring with increasing age. An age-related change of the coupling of neural activity to the BOLD hemodynamic response was described by D'Esposito et al. (1999). Ross and colleagues (1997) also found that the amplitude of the BOLD response was significantly decreased in elderly, as compared to younger subjects, suggesting a reduction in functional activation, or an age-related alteration in the coupling of blood oxygenation to focal activation. These changes were not simply related to increased movement artifacts in elderly subjects.

The observation that some aspects of the hemodynamic coupling between neural activity and BOLD fMRI signals are age dependant cautions against simple interpretations of the results of imaging studies comparing young and elderly subjects. Moreover, our observation, together with the above-mentioned studies, argues for the need of age-matched control examinations, particularly when fMRI is to be routinely applied in the presurgical decision-making. Our results suggest that familial sinistrality does influence the lateralization of language function. This relation was significant for DLT and fMRI. In accordance with Annett (1994), we found that familial LH showed a clearer right-hemispheric involvement as opposed to non-familial LH. The underlying physiology was described by Hecaen et al. (1981), who suggested that familial sinistrality is associated with a more widespread distribution of language functions, resulting in lower LIs. The specialization of the hemispheres could thereby directly be influenced via genetic expression or modulated by several genetic factors.

\section{Contributions from the subdominant hemisphere}

Only one RH and one LH exclusively activated one hemisphere during the word classification task. The vast majority of subjects showed significant activation in both the dominant and subdominant hemispheres. While a more pronounced bilateral recruitment of eloquent cortices would be expected in the group of $\mathrm{LH}$, the presence of right hemisphere language-related activation in nearly all RH is rather intriguing. This observation argues for the view that language lateralization is a continuously variable phenomenon. Categorical designations such as 'left dominant', 'right dominant', and 'bilateral' are nevertheless common and no doubt useful in certain clinical settings. However, the utility of this classification for the understanding of the neurobiology of language organization is questionable. Several authors have proposed that the variability of lateralization assessment may simply be due to differences in categorization methods, i.e., the definition of cut-offs to classify for left, right, and bilateral dominance (Loring et al. 1990; Snyder et al. 1990; Risse et al. 1997). We suggest that the individual LI case can be more valuable in predicting the definite surgical risk than the classification in a global category. However, further research will be necessary to evaluate the importance of subdominant contributions to the global language network, in particular during recovery from brain lesions.

\section{A non-invasive regime for functional decision-making}

Our study demonstrated that fMRI can assess lateralization effects of hemispheric language organization by means of word classification tasks, which mainly result in activation of the inferior frontal gyrus. We used the DLT as an additional tool, which evaluates the perceptive aspect of language function. The global agreement between the two techniques was very high $(97.1 \%)$. Con- 
flicting results or diverging indices were observed in 11 subjects. Increasing age, non-familial sinistrality, and a non-dominant writing hand were identified as the main factors accompanying mismatch between techniques. This finding can be understood as a reflection of an intrahemispheric distribution of language function, different aspects of which are assessed by the two applied techniques.

\section{References}

Annett M (1985) Left, right, hand and brain: the right shift theory. Erlbaum, Hillsdale, NJ

Annett M (1994) Handedness as a continuous variable with dextral shift: sex, generation and family handedness in subgroups of left- and right-handers. Behav Genet 24:51-63

Benbadis SR, Binder JR, Swanson SJ, Fischer M, Hammeke TA, Morris GL, Frost JA, et al (1998) Is speech arrest during Wada testing a valid method for determining hemispheric representation of language? Brain Lang 65:441-446

Binder JR, Swanson SJ, Hammeke TA, Morris GL, Mueller WM, Fischer M, et al (1996) Determination of language dominance using functional MRI: a comparison with the Wada test. Neurology 46:978-984

Buckner RL, Raichle ME, Peterson SE (1995) Dissociation of human prefrontal cortical areas across different speech production tasks and gender groups. J Neurophysiol 74:2163-2173

Cabeza R, McIntosh A, Tulving E, Nyberg L, Grady C (1997) Age-related differences in effective neural connectivity during encoding and recall. Neuroreport 8:3479-3483

Demb J, Desmond J, Wagner A, Vaidya C, Glover G, Gabrieli D (1995) Semantic encoding and retrieval in the left inferior, prefrontal cortex: a functional MRI study of task difficulty and process specificity. J Neurosci 15:5870-5878

Desmond JE, Sum JM, Wagner AD, Demb JB, Shear PK, Glover $\mathrm{GH}$, et al (1995) Functional MRI measurement of language lateralization in Wada-tested patients. Brain 118:1411-1419

D’Esposito M, Zarahn E, Aguirre GK, Rypma B (1999) The effect of normal aging on the coupling of neural activity to the bold hemodynamic response. Neuroimage 10:6-14

Diesch E, Haettig H (1997) Speechlab: PC software for digital speech signal processing. Behav Res Methods Instrum Comput 29:302

Ferstl EC, Cramon DY von (2000) The locus of coherence and cohesion in text comprehension: an event-related fMRI study. CNS, San Francisco

Frost JA, Binder JR, Springer JA, Hammeke TA, Bellgowan PS, Rao SM, Cox RW (1999) Language processing is strongly left lateralized in both sexes. Evidence from functional MRI. Brain 122:199-208

Gabrieli J, Desmond J, Demb JB, Wagner AD, Stone MV, Vaidya C, et al (1995) Functional magnetic resonance imaging of semantic memory processes in the frontal lobes. Psychol Sci $7: 278-283$

Gerschlager W, Lalouschek W, Lehrner J, Baumgartner C, Lindinger G, Lang W (1998) Language-related hemispheric asymmetry in healthy subjects and patients with temporal lobe epilepsy as studied by event-related brain potentials and intracarotid amobarbital test. Electroencephalogr Clin Neurophysiol 108:274282

Hajek M, Valavanis A, Yonekawa Y, Schiess R, Buck A, Wieser H (1998) Selective amobarbital test for the determination of language function in patients with epilepsy with frontal and posterior temporal lesions. Epilepsia 39:389-398
Hecaen H, De Agostini M, Monzon-Montes A (1981) Cerebral organization in left-handers. Brain Lang 12:261-284

Hermans J, Habbema JDF, Kasanmoentalib TKD, Raatgever JW (1982) ALLOC 80: discriminant analysis program. University of Leiden, The Netherlands

Hertz-Pannier L, Gaillard W, Mott S, Cuenod C, Bookheimer S, Weinstein S, et al (1997) Noninvasive assessment of language dominance in children and adolescents with functional MRI. Neurology 48:1003-1011

Hongkeun K (1994) Distribution of hemispheric asymmetry in left-handers and right-handers: data from perceptual asymmetry studies. Neuropsychology 8:148-159

Hugdahl K (2000) Lateralization of cognitive processes in the brain. Acta Psychol 105:211-235

Hugdahl K, Bronnick K, Kyllingsbaek S, Law I, Gade A, Paulson OB (1999) Brain activation during dichotic presentations of consonant-vowel and musical instrument stimuli: a 150-PET study. Neuropsychologia 37:431-440

Kimura D (1967) Functional asymmetry of the brain in dichotic listening. Cortex 3:163-178

Kruggel F, Lohmann G (1996) BRIAN (brain image analysis): a toolkit for the analysis of multimodal brain datasets. In: Lemke HU, Vannier M, Inamura K, Farman A (eds) Computer assisted radiology. Proc Int Symp Computer and Communication Systems for Image Guided Diagnosis and Therapy. Elsevier, Amsterdam, pp 323-328

Lee G, Loring D, Newell J, Meador K (1994) Is dichotic word listening a valid predictor of cerebral language dominance? Clin Neuropsychol 8:429-438

Lehéricy S, Cohen L, Bazin B, Samson S, Giacomini E, Rougetet R, Hertz-Pannier L, Le Bihan D, Marsault C, Baulac M (2000) Functional MR evaluation of temporal and frontal language dominance compared with the Wada test. Neurology 54:16251633

Loring D, Meador K, Lee G, Murro A, Smith J, Flanigin H, et al (1990) Cerebral language lateralization: evidence from intracarotid amobarbital testing. Neuropsychologia 28:831-838

Oldfield R (1971) The assessment and analysis of handedness: the Edinburgh inventory. Neuropsychologia 9:97-113

Price CJ, Moore CJ, Friston KJ (1996) Getting sex into perspective. Neuroimage 3:586

Risse G, Gates J, Fangman M (1997) A reconsideration of bilateral language representation based on the intracarotid amobarbital procedure. Brain Cogn 33:118-132

Ross MH, Yurgelun-Todd DA, Renshaw PF, Maas LC, Mendelson JH, Mello NK, et al (1997) Age-related reduction in functional MRI response to photic stimulation. Neurology 48:173-176

Shaywitz B, Shaywitz S, Pugh K, Constable R, Skudlarsky P, Fulbright R, et al (1995) Sex differences in the functional organization of the brain for language. Nature 373:607-609

Snyder PJ, Novelly RA, Harris LJ (1990) Mixed speech dominance in the intracarotid sodium amytal procedure: validity and criteria issues. J Clin Exp Neuropsychol 12:629-643

Springer JA, Binder J, Hammeke T, Swanson S, Frost J, Bellgowan $P$, et al (1999) Language dominance in neurologically normal and epilepsy subjects. Brain 122:2033-2045

Strauss E, Gaddes W, Wada J (1987) Performance on a free-recall verbal dichotic listening task and cerebral dominance determined by the carotid amytal test. Neuropsychologia 25:747753

Talairach P, Tournoux J (1988) A stereotactic coplanar atlas of the human brain. Thieme, Stuttgart

Wada J, Rasmussen T (1960) Intracarotid injection of sodium amytal for the lateralization of cerebral speech dominance. J Neurosurg 17:266-282

Zatorre R (1989) Perceptual asymmetry on the dichotic fused words test and cerebral speech lateralization determined by the carotid sodium amytal test. Neuropsychologia 27:1207-1219 